\title{
Application of an improved PSO algorithm mixed BFO algorithm in optimal scheduling of hydropower station
}

\author{
Yibo Zou ${ }^{1}, \mathrm{Li} \mathrm{Mo}^{1, \mathrm{a}}$, Xiaogang Xiao ${ }^{2}$ \\ ${ }^{1}$ Shcool of Hydropower and Information Engineering, Huazhong University of Science and Technology, Wuhan, Hubei 430074, China \\ ${ }^{2}$ Central China Power Grid Company, Wuhan, Hubei 430077, China
}

\begin{abstract}
The optimal scheduling of hydropower station is a constrained strong, nonlinear and multi-stage combinatorial optimization. Aiming at this issue, this paper analyses the shortcomings of previous PSO algorithm in hydropower station optimal scheduling model, and presents an improved PSO algorithm for hybrid BFO algorithm, which overcomes the problem that the PSO algorithm is easy to fall into local extremum and has strong dependence on parameters. A case study of a short-term scheduling period of a hydropower station is used to compare the improved PSO algorithm mixed BFO algorithm with previous PSO algorithm. The results show that the improved PSO algorithm can converge to the global optimal solution more accurately. Therefore, it provides a new method for solving the optimal scheduling model of hydropower station.
\end{abstract}

\section{Introduction}

With the development of hydropower industry, it is a hotspot to solve the problem of optimal scheduling of hydropower stations by using systematic and scientific methods. At present, the basic method to solve the optimal scheduling problem of hydropower stations is to establish an optimal scheduling model for hydropower stations. Dynamic programming algorithm [1] (DP), Genetic algorithm (GA), Progress optimality algorithm (POA), Particle swarm optimization algorithm ${ }^{[2]}$ (PSO) are used to solve this model. These algorithms ${ }^{[3]}$ have their own advantages and disadvantages. The PSO algorithm is an evolutionary algorithm. Starting from the random solution and finding the optimal solution by iteration, it has the advantages of easy implementation, high precision and fast convergence. Therefore, it is widely used in the hydropower station optimization scheduling model ${ }^{[4]}$. However, at the same time, there is a problem that the convergence speed is fast but it is easy to fall into the local extremum and has strong dependence on the parameters, and the BFO has the characteristics of strong global search ability but low efficiency. This paper introduces the characteristics of the BFO algorithm, and updates the particle position by using the trending step of the BFO algorithm in the PSO algorithm after the updated fitness value is smaller than that of the previous generation. An improved PSO algorithm based on hybrid BFO algorithm is proposed, which overcomes the limitations of PSO algorithm and provides a new method for solving hydropower station optimal scheduling model.

\section{PSO algorithm and Improved PSO algorithm}

\subsection{PSO algorithm}

Particle swarm optimization (PSO) is an evolutionary computation that was developed in 1995 by Dr. Eberhart and Dr. Kennedy from a behavioral study of predation of birds. The algorithm was originally inspired by the regularity of the bird cluster activity, and then a simplified model built using group intelligence. Based on the observation of the activity behavior of animal clusters, the PSO algorithm uses the individual's information sharing in the group to make the whole group's motion generate the disordered to ordered evolution process in solving the space optimal, and obtain the optimal solution.

Initialize a group of random particle swarms (random solutions) of a number $\mathrm{m}$ in a $\mathrm{D}$-dimensional space, each particle representing a feasible solution, where the position of the $\mathrm{i}$-th particle in the D-dimensional search space is represented as a vector $X=\left(x_{i 1}, x_{i 2}, \ldots, x_{i D}\right)^{T}$, its "flying" speed is represented as $V=\left(v_{i 1}, v_{i 2}, \ldots, v_{i D}\right)^{T}$.In each iteration, the particle updates itself by tracking two "extreme values". The first is the optimal solution found by the particle itself. This solution is called the individual extremum (Pbest). The other extreme is the optimal solution currently found for the entire population. This extreme is the global extremum (Gbest). Alternatively, instead of the entire population and only a part of it as a neighbor of the

*Corresponding author: moli@hust.edu.cn 
particle, the extremum in all neighbors is the local extremum.

When you find these two optimal values, the particles update their speed and position according to the following formula:

$$
\begin{gathered}
V_{i d}^{k+1}=\omega V_{i d}^{k}+c_{1} r_{1}^{k}\left(P_{d}^{k}-X_{i d}^{k}\right)+c_{2} r_{2}^{k}\left(G_{d}^{k}-X_{i d}^{k}\right) \\
X_{i d}=X_{i d}+V_{i d}
\end{gathered}
$$

Where $i=(1,2, \ldots, m), d=(1,2, \ldots, D) ; \omega$ denotes Non-negative constant, called as Inertia factor, $\omega$ can also be linearly reduced with iteration, and the value is generally between $[0.8,1.2] ; c_{1}, c_{2}$ denotes learning factor non-negative constant; $r_{1}, r_{2}$ denotes random number between $[0,1] ; V_{i d} \in\left(-V_{\max }, V_{\max }\right)$;

\subsection{Improved PSO algorithm}

The bacterial foraging algorithm was proposed by K.M. Passino in 2002 based on the behavior of Ecoli E. coli in the human intestine to phagocytose food. The algorithm has the advantages of group parallel search and easy to jump out of local minimum values. The chemotactic process is to simulate the movement of a large intestine cell, by using the flagella to swim and flip. In biology, an E. coli can move in two different ways. It can swim in the same direction for a certain period of time or can be flipped, or alternate between the two modes of operation throughout the life cycle. In the process of calculating the trend, the direction of movement of bacteria may be expressed as follows:

$$
\theta^{i}(j+1, k, l)=\theta^{i}(j, k, l)+C(i) \frac{\Delta(i)}{\sqrt{\Delta^{T}(i) \Delta(i)}}
$$

Where $\Delta$ denotes a unit vector in the random direction, which is a step size, $\theta^{i}(j+1, k, l)$ denotes the position of the particle. PSO algorithm is easy to fall into local optimum and miss the optimal solution in the later stage $^{[5]}$. Adding the chemotaxis step of BFO algorithm can increase the diversity of particle search. The essence of the idea is to change the particles with smaller adaptive values in the iterative process of particle swarm. The location makes its search jump out of local optimum, which enhances the global search ability.

The specific steps of the improved PSO algorithm are as follows:

Step 1: Initialize a group of particles of the number $m$ in the D-dimensional space, initialize the current evolution algebra $k=1$, and set $K_{\max }$ as the largest evolution algebra.

Step 2: Obtain the current position information of the particle and substitute it into the fitness function to calculate the current adaptive value. Each particle is an individual optimal particle(Pbest). Then traverse the size of the fitness value between the particles, and the particle with the largest fitness value is taken as the global optimal particle(Gbest).

Step 3: The velocity and position of the particles are updated by formulas (1) and (2). The fitness values of each updated particle are compared and the fitness values of the previous generation particles are traversed. The particles with increased fitness value are directly subjected to step 4; The reduced-valued particles change their position according to equation (3), and then proceed to step 4 .

Step 4: Select the particle with the largest fitness value in the group and compare it with Gbest. If it is larger than Gbest, replace it. Compare the size of each particle to Pbest, and replace it if it is larger than Pbest.

Step 5: When the number of iterations reaches the maximum number of iterations, the program ends. At this time, the particle information is the global optimal solution; otherwise, the order returns to step 3.

\section{SCHEDULING MODEL}

In the process of inner diameter flow in a given control period, under the comprehensive utilization requirements of hydropower stations and other constraints, the mathematical model of optimal operation of hydropower station is established with the maximum power generation as the objective function.

\section{1 objective function}

$$
\max N=\sum_{i=1}^{T} A Q \Delta H
$$

Where $N$ denotes hydropower station generates electricity during the total period; $T$ denotes number of time periods divided during the total period; $A$ denotes comprehensive output coefficient, Generally taken as 8.5; $Q$ denotes power generation flow, According to the initial water level $Z_{i}$ and the final water level $Z_{f}$ of corresponding time period $t$, inflow $I$, the relationship between water level and storage capacity came to determine. The specific process is to query the relationship between water level and storage capacity to obtain the initial storage volume $V_{i}$ and the final storage volume $V_{f}, Q$ is calculated from the flow balance formula; $\Delta H$ denotes water head, is the difference between the upstream average water level and the downstream average water level during this period. The upstream average water level is the average of $Z_{i}$ and $Z_{f}$, The downstream water level is determined by discharge.

\section{2 constraint condition}

(1) Water balance equation

$$
Q_{t}=I-\left(V_{i}-V_{f}\right) / t
$$


Where $I$ and $Q_{t}$ denote the inflow and outflow in period $t ; V_{i}$ and $V_{f}$ denote the initial water storage volume and the final storage volume in period $t$.

(2) Water level constraints

$$
Z_{t, \min } \leq Z_{t} \leq Z_{t, \max }
$$

Where $Z_{t, \min }$ and $Z_{t, \max }$ denote the minimum and maximum water level limits in period $t$.

(3) Output constraints

$$
N_{b t} \leq N_{t} \leq N_{y t}
$$

Where $N_{b t}$ and $N_{y t}$ denote guaranteed output and expected output of hydropower stations in period $t$.

(4) Water discharge constraints

$$
Q_{t, \text { min }} \leq Q_{t} \leq Q_{t, \text { max }}
$$

Where $Q_{\mathrm{t}, \min }$ and $Q_{\mathrm{t} \text {,max }}$ denote the maximum and minimum water discharge limits in period $t$.

\section{CASE STUDY}

In order to verify the effectiveness of the improved PSO algorithm in practical applications, this paper takes the optimal scheduling of a hydropower station as an example. It is known that the dead water level of hydropower station and the maximum limit water level during the dispatching period are $190 \mathrm{~m}$ and $200 \mathrm{~m}$ respectively; the maximum water discharge and minimum water discharge are $1292 \mathrm{~m}^{3} / \mathrm{s}$ and $73 \mathrm{~m}^{3} / \mathrm{s}$ respectively; the installed capacity of the power station is 1.2 million $\mathrm{KW}$; The relationship between water level and reservoir capacity and the relationship between downstream water level and discharge are also known.

The daily average inflow and the initial-final water level of the target hydropower station are selected as input conditions. The improved PSO algorithm is used to solve the optimal hydropower station scheduling model. At the same time, the PSO algorithm is used to solve the optimal hydropower station scheduling model. Obtain the water level change process, the output and cumulative output process in each period of the hydropower station under the two algorithms. When calculating the model, the relevant parameters of the improved PSO algorithm are set as follows: $\omega=0.8$; $c_{1}=c_{2}=2 ; m=50 ; K_{\max }=100$ 。

The calculation result of the improved PSO algorithm in the example is compared with the calculation result of the PSO algorithm in the example: It can be seen from Tab1 and Fig $1^{[6]}$ that under the constraints of hydropower station. The total output of the improved PSO algorithm is greater than the PSO algorithm, and the difference between highest and lowest water level of improved PSO algorithm is larger than the PSO algorithm, which indicates that the improved PSO algorithm has a wider range of search in the global scope and the final fitness value is larger.
Table 1. Comparison of the output between PSO algorithm and improved PSO algorithm

\begin{tabular}{|c|c|c|c|}
\hline Algorithm & $\begin{array}{c}\text { Accumulative } \\
\text { power(MW) }\end{array}$ & $\begin{array}{c}\text { Minimu } \\
\mathrm{m}(\mathrm{MW})\end{array}$ & $\begin{array}{c}\text { Maximum } \\
\text { (MW) }\end{array}$ \\
\hline PSO & 12488 & $71 \mathrm{r}$ & 1241 \\
\hline PSO-BFO & 12722 & 71 & 1116 \\
\hline
\end{tabular}

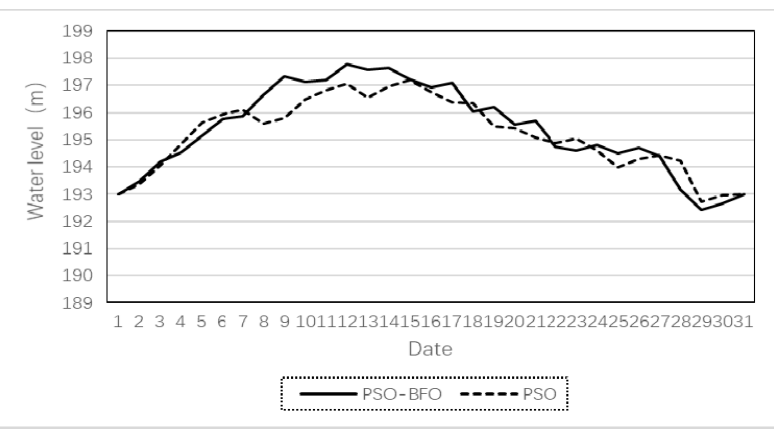

Fig.1 Upstream water level change process line diagram of improved PSO algorithm and PSO algorithm

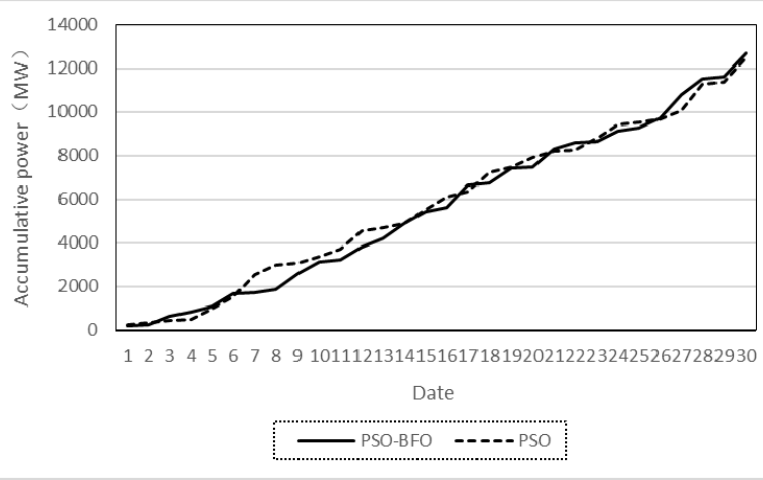

Fig.2 Cumulative output process diagram of improved PSO algorithm and PSO algorithm

\section{CONCLUSIONS}

As a method to solve the optimal scheduling model of hydropower station, PSO algorithm has its advantages, but it has the disadvantage of being easily trapped in local optimum. The chemotaxis step introduced in the BFO algorithm overcomes this shortcoming well and can obtains better particle information. The example proves that the improved PSO algorithm can better solve the multi-dimensional strong constraint, multi-stage and nonlinear combination problems in hydropower station optimization scheduling, and strengthen the solving ability, which provides an effective method for hydropower station optimization scheduling.

Acknowledgement This work was supported by the National Key R\&D Program of China (No. 2016YFC0402210) and the National Natural Science Foundation of China (No. 51479075), the Fundamental Research Funds for the Central Universities ( No. 2017KFYXJJ199) and the Science and Technology Project of State Grid Corporation (No. SGHZ0000DKJS1800195). 


\section{ACKNOWLEDGEMENT}

This work was supported by the National Key R\&D Program of China (No. 2016YFC0402210) and the National Natural Science Foundation of China (No. 51479075), the Fundamental Research Funds for the Central Universities (No. 2017KFYXJJ199) and the Science and Technology Project of State Grid Corporation (No. SGHZ0000DKJS1800195).

\section{References}

1. Allen RB, Bridgeman SG. Dynamic programming in hydropower scheduling. J Water Resour Plan Manage 1986;112(3):339-53.

2. Cheng CT, Liao SL, Tang ZT, and Zhao MY. Comparison of particle swarm optimization and dynamic programming for large scale hydro unit load

dispatch[J].EnergyConversionandManagement,2009 ,50(12).LuigiT.DeLuca, Propulsion physics (EDP Sciences, Les Ulis, 2009)

3. Xie W, Ji CM., Yang ZJ, and Zhang XX. Short-term power generation scheduling rules for cascade hydropower stations based on hybrid algorithm. 2012. Water Science and Engineering, 5(1), 46-58. [doi:10.3882/j.issn.1674-2370. 2012.01.005]

4. Fan YR, Huang W, Huang GH, Huang K, Zhou X (2015) A PCM-based stochastic hydrological model for uncertainty quantification in watershed systems. Stoch Env Res Risk A 29:915-927.

5. Zhang GS; Su Y Application of Improved Particle Swarm Optimization Algorithm in Medium and Long Term power Load Combination Forecasting.2016. Proceedings of 2016 2nd International Conference on Economics,Management Engineering and Education Technology

6. Yang ZJ, Wang LP , Shao L, and Wu YQ. Drawing of the reservoir operation chart based on theparticle swarm optimization algorithm. 2010. Power System Protection and Control, 38(14), 59-62. 